\title{
The utility of a portable sleep monitor to diagnose sleep-disordered breathing in a pediatric population
}

\author{
Colin Massicotte RPSGT ${ }^{1}$, Suhail Al-Saleh MBBS FRCPC MSc ${ }^{1,2}$, \\ Manisha Witmans MD FRCPC FAASM ${ }^{3}$, Indra Narang BMedSci MBBH MD ${ }^{1,2}$
}

C Massicotte, S Al-Saleh, M Witmans, I Narang. The utility of a portable sleep monitor to diagnose sleep-disordered breathing in a pediatric population. Can Respir J 2014;21(1):31-35.

BACKGROUND: Central and/or obstructive sleep-disordered breathing (SDB) in children represents a spectrum of abnormal breathing during sleep. SDB is diagnosed using the gold standard, overnight polysomnography (PSG). The limited availability and access to PSG prevents its widespread use, resulting in significant delays in diagnosis and treatment of SDB. As such, portable sleep monitors are urgently needed.

OBJECTIVE: To evaluate the utility of a commercially available portable sleep study monitor (PSS-AL) (ApneaLink, ResMed, USA) to diagnose SDB in children.

METHODS: Children referred to a pediatric sleep facility were simultaneously monitored using the PSS-AL monitor and overnight PSG. The apnea-hypopnea index (AHI) was calculated using the manual and autoscoring function of the PSS-AL, and PSG. Sensitivity and specificity were compared with the manually scored PSS-AL and PSG. Pearson correlations and Bland-Altman plots were constructed.

RESULTS: Thirty-five children (13 female) completed the study. The median age was 11.0 years and the median body mass index z-score was 0.67 (range -2.3 to 3.8 ). SDB was diagnosed in 17 of 35 (49\%) subjects using PSG. The AHI obtained by manually scored PSS-AL strongly correlated with the AHI obtained using PSG $(r=0.89 ; \mathrm{P}<0.001)$. Using the manually scored PSS-AL, a cut-off of AHI of $>5$ events/h had a sensitivity of $94 \%$ and a specificity of $61 \%$ to detect any SDB diagnosed by PSG.

CONCLUSIONS: Although PSG is still recommended for the diagnosis of SDB, the ApneaLink sleep monitor has a role for triaging children referred for evaluation of SDB, but has limited ability to determine the nature of the SDB.

Key Words: Children; Polysomnography; Portable monitoring; Sleep-disordered breathing

Sleep-disordered breathing (SDB) represents a group of respiratory $\checkmark$ disorders specific to sleep or exacerbated by sleep that include obstructive sleep apnea (OSA), central sleep apnea, periodic breathing and hypoventilation. The most common sleep disorder in children is OSA, characterized by snoring; recurrent, partial (ie, hypopneas) or complete obstruction (ie, apneas) of the upper airway; intermittent oxyhemoglobin desaturations; and sleep disruption. OSA affects $1 \%$ to $4 \%$ of all healthy children (1), with peak presentation at two to eight years of age, coinciding with adenotonsillar hypertrophy, the most common etiological factor for OSA (1). More recently, the epidemic of obesity has highlighted childhood obesity as a significant risk factor for OSA, with a reported prevalence of up to $60 \%$ (2). Central sleep apnea, periodic breathing or central hypoventilation are less common than OSA, and can be classified as primary (eg, congenital central hypoventilation syndrome) or secondary (eg, Arnold-Chiari malformations) (3).

The gold standard test to diagnose SDB is polysomnography (PSG), which is an important tool to differentiate the various types of SDB $(4,5)$. There is currently an unprecedented increase in the

\author{
L'utilité d'un moniteur portable du sommeil pour \\ diagnostiquer les troubles respiratoires du sommeil \\ dans une population d'âge pédiatrique
}

HISTORIQUE : Les troubles respiratoires du sommeil (TRS) chez les enfants, qu'ils soient centraux ou obstructifs, représentent un spectre de respiration anormale pendant le sommeil. Les TRS sont diagnostiqués au moyen du test de référence, la polysomnographie d'une nuit (PSG). La disponibilité et l'accès limités de la PSG en empêchent l'utilisation généralisée, ce qui entraîne d'importants délais dans le diagnostic et le traitement des TRS. C'est pourquoi des moniteurs portables du sommeil s'imposent de toute urgence.

OBJECTIF : Évaluer l'utilité d'un moniteur portable d'étude du sommeil (MPS-AL) en vente libre (ApneaLink, ResMed, États-Unis) pour diagnostiquer les TRS chez les enfants.

MÉTHODOLOGIE : Les enfants aiguillés vers un centre pédiatrique des troubles du sommeil ont subi une surveillance simultanée à l'aide du MPS-AL et de la PSG d'une nuit. Les chercheurs ont calculé l'index d'apnées-hypopnées (IAH) au moyen de la fonction manuelle et automatique du MPS-AL, ainsi que de la PSG. Ils ont comparé la sensibilité et la spécificité avec le MPS-AL obtenu manuellement et avec la PSG. Ils ont construit des corrélations de Pearson et des graphiques de Bland-Altman. RÉSULTATS : Trente-cinq enfants (13 filles) ont terminé l'étude. Ils avaient un âge médian de 11,0 ans et un écart réduit médian de l'indice de masse corporelle de 0,67 (plage de 2,3 à 3,8). On a diagnostiqué des TRS chez 17 (49 \%) d'entre eux au moyen de la PSG. L'IAH obtenu par la fonction manuelle du MPS-AL était fortement corrélé avec l'IAH obtenu au moyen de la PSG $(r=0,89 ; \mathrm{P}<0,001)$. Au moyen de la fonction manuelle du MPS-AL, un seuil d'IAH de plus de 5 événements/h permettait de déceler les TRS diagnostiqués par PSG selon une sensibilité de $94 \%$ et une spécificité de $61 \%$.

CONCLUSIONS : Même si la PSG est toujours recommandée pour diagnostiquer les TRS, le moniteur du sommeil ApneaLink joue un rôle dans le triage des enfants aiguillés en vue d'une évaluation des TRS, mais est peu utile pour déterminer la nature des TRS.

demand for testing for SDB due to an increasing awareness of pediatric sleep disorders, in particular, obesity-related OSA; the recognition of adverse consequences of SDB on cardiovascular, metabolic and neurobehavioural functions as well as the quality of life of children with SDB (6-10); and recommendations for preoperative monitoring with PSG for adenotonsillectomy surgery (10-12).

The prevalence of SDB apparent across both the developmental spectrum and disease pathways produces no specific constellation of symptoms that is sufficiently sensitive to predict the presence of SDB in all children. OSA has several modifiable risk factors for which prompt diagnosis and management may prevent comorbidity associated with OSA. However, it is evident that the demand for pediatric PSG cannot be met (13) and alternative tools, including questionnaires (14,15), videos (16) and overnight oximetry (17), have been explored with limited success. In one centre, an abnormal pulse oximetry had a positive predictive value of $95 \%$ in determining OSA; however, many of the children in that study had normal oximetry and required formal PSG or repeat oximetry (17).

${ }^{1}$ Division of Respiratory Medicine, The Hospital for Sick Children; ${ }^{2}$ University of Toronto, Toronto, Ontario; ${ }^{3}$ University of Alberta, Edmonton, Alberta

Correspondence: Dr Indra Narang, Division of Respiratory Medicine, The Hospital for Sick Children, 555 University Avenue, Toronto,

Ontario M5G 1X8. Telephone 416-813-6346, fax 416-813-6246, e-mail indra.narang@sickkids.ca 
Given these limitations, a portable sleep monitor may be useful to screen and triage more medically urgent patients. The role of a portable sleep study (PSS) in children, either as a screening or diagnostic tool, has not been fully explored despite the fact that pediatric guidelines suggest that when a PSG is unavailable, other options, such as portable monitoring, should be used to best determine the likelihood of OSA (18).

One such PSS device, the ApneaLink (AL) monitor (ResMed, USA) has been validated in adults with a high sensitivity and specificity (19-21). The differences in the pathophysiology and management of SDB in children compared with adults does not allow for adult-specific data to simply be extrapolated to children. Recently, the AL monitor was evaluated in 25 obese adolescents (mean age 13 years) simultaneously with overnight PSG in a sleep laboratory and yielded a sensitivity and specificity of $85 \%$ and $83 \%$, respectively, for apnea-hypopnea index (AHI) $>5$ events/h (22). However, the investigators did not include younger children, who are typically the more common at-risk age group for SDB.

The aim of the present study was to evaluate the utility of the AL monitor as a PSS for the diagnosis of SDB in children who typically present to a pediatric sleep facility. We hypothesized that the AL monitor could help in identifying children with moderate to severe SDB.

\section{METHODS}

Children four to $<18$ years of age with a history of snoring who were referred for an initial baseline PSG at The Hospital for Sick Children, Toronto, Ontario, from 2008 to 2010, were consecutively recruited for the present study. The Hospital for Sick Children is a tertiary referral centre and is the only designated pediatric sleep facility covering a large radius and, thus, receives many community referrals. Approximately $75 \%$ of referred children have underlying comorbidities. Children were excluded if they had previously received intervention before or during PSG (eg, adenotonsillectomy for OSA, oxygen or positive airway pressure therapy) or if they had ever had a PSG diagnosis of OSA. Children with known neurodevelopmental disorder were also excluded due to the lack of tolerability of PSG equipment, the burden of which would be increased with simultaneous PSS-AL monitoring.

The Research Ethics Board at The Hospital for Sick Children reviewed and approved the protocol, and subjects provided consent and assent before participating in the study.

\section{Overnight PSG}

All PSG studies were reported by pediatric sleep physicians who were blinded to the results of the PSS-AL. Patients underwent standard overnight PSG using a data acquisition and analysis system (XLTEK, Natus Medical Inc, USA). Respiratory measurements included chest wall and abdominal movements using chest wall and abdominal belts; nasal airflow measurements using nasal air pressure transducer and nasal thermal sensor; oxygen saturation using oximeter (Masimo Corporation, USA), transcutaneous carbon dioxide $\left(\mathrm{tcCO}_{2}\right)$ and endtidal carbon dioxide $(\mathrm{etCO} 2$ ). The PSG system used Braebon (Braebon Medical Corporation, Canada) QRIP respiratory inductive plethysmography effort sensors, cannula connected to Braebon pressure transducer and Braebon Thermistor. The $\mathrm{tcCO}_{2}$ was recorded using Linde Microgas 7650 (Basel, Switzerland) and etCO $\mathrm{CO}_{2}$ was recorded using a capnograph (Capnocheck Sleep, BCI, USA). PSG measurements also included electroencephalogram, electro-oculogram, and submental and bilateral anterior tibialis electromyograms. Video and audio recordings were obtained for each study and body position. Sleep architecture was assessed using standard techniques (23). Information obtained from PSG included sleep-onset latency and rapid eye movement (REM) onset latency, total sleep time (TST), sleep efficiency, time spent in each sleep stage (minutes and percentage), number and classification of arousals, number of independent leg movements and snoring. Recorded respiratory data included counts and indexes of the following events: obstructive apneas, obstructive hypopneas, central apneas and mixed apneas. All events were scored according to the American Academy of Sleep Medicine (AASM) scoring guidelines for children (23) by a registered PSG technician who was blinded to the results of the PSS-AL. An obstructive apnea event was scored when airflow decreased $>90 \%$ from baseline for at least $90 \%$ of the entire respiratory event with chest and/or abdominal motion throughout the entire event, the duration of which was at least a minimum of two baseline breaths. A hypopnea event was scored when airflow dropped by at least $50 \%$ from baseline, the duration of which was at least a minimum of two baseline breaths. The hypopnea event must have been accompanied by a minimum 3\% drop in oxygen desaturation, an arousal or an awakening (23). A central apnea event was defined as a cessation of airflow with an absence of respiratory and abdominal effort for a minimum of $20 \mathrm{~s}$ or of the duration of at least two baseline breaths, in which case the event must be accompanied by a minimum $3 \%$ drop in oxygen desaturation, an arousal or an awakening. Central hypopneas were not evaluated. A mixed apnea event was scored when airflow decreased $>90 \%$ from baseline for at least $90 \%$ of the entire respiratory event, the duration of which was a minimum of two baseline breaths, which is associated with absent inspiratory effort in the initial portion of the event, followed by resumption of inspiratory effort before the end of the event. SDB severity was graded according to accepted clinical criteria. The AHI was the number of obstructive apneas, hypopneas, mixed apneas and central apneas per hour during sleep. An AHI <1.5 events/h was considered to be normal (24); an AHI from 1.5 events/h to $<5$ events/h was considered to be mild SDB; an AHI from 5 events/h to $<10$ events/h was considered to be moderate SDB; and an AHI $\geq 10$ events/h was considered to be severe SDB.

\section{AL monitor}

The PSS monitor used was the AL device, which consists of a nasal cannula attached to a small case that houses a pressure transducer that monitors airflow. The monitor is held in place by a securing belt (the belt plays no role in the device monitoring). Pulse oximetry is also recorded via a digital probe with an averaging time of $1 \mathrm{~s}$, which also monitors heart rate. Children who had $<4 \mathrm{~h}$ of recorded data on the PSS-AL were withdrawn to prevent misclassification. Scoring of the PSS-AL was performed using the proprietary automated software algorithm (provided concurrently with AL). Automated software analysis used the following definitions: an apnea was defined as $\leq 20 \%$ flow relative to baseline; and a hypopnea was defined as $\leq 50 \%$ flow relative to baseline with $3 \%$ desaturation. The duration of each was based on overall computed baseline respiratory rate. The device does not account for mouth breathing and, thus, events are assumed to be apneas when flow limitation occurs. The device is able to identify the presence of a poor airflow signal and this was evaluated. The AL software also enables the investigator to undertake manual scoring, which was performed by a registered PSG technologist who was blinded to the results of the autoscore, clinical history and the overnight PSG results. Manual scoring of apneas and hypopneas used the 2007 AASM pediatric criteria as outlined above. The event classification was determined a priori by the study team. All respiratory events monitored by the PSS-AL were combined to derive an AHI, which was compared with the AHI obtained from PSG.

The present study was unfunded; however, the manufacturer (ResMed, USA) donated the device for the study and associated supplies. ResMed was not involved in any data collection, analyses or review of the manuscript.

\section{Statistical analysis}

A sample size of 25 was needed to detect a correlation coefficient of 0.60 , with a two-tailed $\alpha=0.05$ and $\beta=0.10$. The analyses were performed using SAS version 9.2 (SAS Inc, USA). Descriptive statistics, including frequencies, percentages, mean, median and/or range values, were obtained for all baseline demographics and PSG data. Frequency statistical analyses were used to observe the prevalence of SDB in this population. The PSS-AL monitor results were compared with the 
reference standard PSG using Fisher's exact test for the classification of SDB severity. Sensitivity, specificity, and positive and negative predictive values for the manually scored PSS-AL were calculated using AHI cut-off values of $\geq 1.5$ events $/ \mathrm{h}$ and $\geq 5$ events $/ \mathrm{h}$. Median values are reported because the data were not normally distributed.

Bland-Altman plots were used as a graphical representation of the observed differences between the paired measurements to examine the relationship between the magnitude and degree of variation in the AHI measurements using full PSG and the PSS-AL. Correlations between the AHI obtained from PSG and the manually scored PSS-AL, and between the automated and manual PSS-AL AHI were determined using Pearson's correlation coefficient and intraclass correlation.

\section{RESULTS}

Forty-five subjects enrolled in the study, of which complete data for the PSS-AL and PSG were available for 35 (13 female). Overall, 10 of $45(22 \%)$ studies had to be excluded because of limited recording time $(<4$ h of TST) or because there was insufficient flow to accurately determine respiratory events. The loss of flow data, either due to artefact or the displacement of the nasal cannula, was 19\% across the flow signals of all the children who were included in the study. The median age of the subjects was 11.0 years and the median body mass index (BMI) z-score was 0.67 (Table 1), with 10 of 35 children classified as obese, which was defined as a BMI $z$-score $\geq 1.65$. Underlying comorbidities of the subjects included sickle-cell disease $(n=13)$; central nervous system tumours $(n=3)$ and Arnold-Chiari malformation $(n=2)$. The median AHI of the subjects was 1.2 events/h (range 0.0 to 50.8 events/h). There were no significant differences between patients with comorbidities and healthy children in terms of age, sex, BMI, snoring and AHI. The median sleep latency and REM latency were $14 \mathrm{~min}$ and $155 \mathrm{~min}$, respectively. The median TST was $373 \mathrm{~min}$ and the percentage of TST in stage 1, 2, 3 and REM sleep were 4.6\%, 51.2\%, $28.2 \%$ and $15.6 \%$, respectively.

Using an AHI of $\geq 1.5$ events/h to diagnose SDB, 18 of 35 (51\%) subjects were classified with SDB and, of these, five of 17 (29\%) had moderate to severe SDB (Table 2). There was a significant correlation between the AHI obtained by manual scoring of the PSS-AL and the PSG AHI (Pearson correlation $\mathrm{r}=0.89 ; \mathrm{P}<0.001$; intraclass correlation $\mathrm{r}=0.81$ [Figure 1]). There was also a significant correlation between manually scored PSS-AL AHI and PSS automated scored AHI (Pearson correlation $\mathrm{r}=0.59 ; \mathrm{P}=0.002$; intraclass correlation $\mathrm{r}=0.57$, [Figure 2]). The correlation between the automated PSS-AL AHI score and the PSG AHI was moderate (Pearson correlation $\mathrm{r}=0.36$; $\mathrm{P}=0.03)$. Using a cut-off $\mathrm{AHI} \geq 1.5$ events $/ \mathrm{h}$, the manually scored PSS-AL monitor had a sensitivity of $94 \%$ and specificity of $16 \%$ to detect any SDB (AHI $\geq 1.5$ events/h) diagnosed by PSG (Table 3). Using a cut-off AHI of $\geq 5$ events/h, the manually scored PSS-AL monitor had a sensitivity of $94 \%$ and specificity of $61 \%$ to detect any SDB (AHI $\geq 1.5$ events/h) diagnosed by PSG and a sensitivity of $100 \%$ with a specificity of $40 \%$ to detect moderate to severe SDB diagnosed by PSG (Table 3). The Bland-Altman plot showed fair agreement between PSS-AL and PSG, with a mean difference of \pm 3.9 events $/ \mathrm{h}$ (Figure 3). Of interest, one of the outliers experienced a large number of hypopneas and apneas recorded on the PSS-AL while he had extended periods of wakefulness documented on the PSG. The second outlier was a 14-year-old female with sickle-cell disease who experienced hypopneas almost exclusively associated with arousals and very few desaturations, accounting for the lower PSS-AL AHI (5.0 events/h) compared with the PSG (10.4 events/h).

\section{DISCUSSION}

The principal findings of the present study show that the PSS-AL monitor can identify SDB in children. The present study found that, using an AHI cut-off value of $\geq 5$ events/h on the manually scored PSS-AL, there was excellent sensitivity and moderate specificity for the diagnosis of SDB compared with PSG. The AL has been used for

\begin{tabular}{|c|c|}
\hline Age, years & $11.0(4.0-17.0)$ \\
\hline Male:female, n:n & $22: 13$ \\
\hline Weight, kg & $43(17.0-145.0)$ \\
\hline Height, cm & $145(54.0-181.0)$ \\
\hline Body mass index, $\mathrm{kg} / \mathrm{m}^{2}$ & $18.1(14.5-42.5)$ \\
\hline Body mass index z-score & $0.67(-2.3$ to +3.8$)$ \\
\hline History of snoring, n (\%) & $24(69)$ \\
\hline Sleep efficiency, \% & $86.8(58.7-96.1)$ \\
\hline Mean $\mathrm{SaO}_{2}, \%$ & $97(89-99)$ \\
\hline Minimum $\mathrm{SaO}_{2}, \%$ & $93(74-96)$ \\
\hline Highest tcCO $/ \mathrm{etCO}_{2}, \mathrm{mmHg}$ & $43(37-55)$ \\
\hline PSG, obstructive apnea-hypopnea index & $0.9(0.1-45.8)$ \\
\hline PSG, central apnea index & $0.1(0.0-9.7)$ \\
\hline PSG, apnea-hypopnea index & $1.2(0.0-50.8)$ \\
\hline PSS-AL (manual scoring), apnea-hypopnea index & $6.0(1.0-48)$ \\
\hline PSS-AL (automated scoring), apnea-hypopnea index & $8.0(1.0-25)$ \\
\hline
\end{tabular}

Data presented as median (range) unless otherwise indicated. et $\mathrm{CO}_{2}$ Endtidal carbon dioxide; PSG Polysomnography; PSS-AL Portable sleep studyApneaLink (ResMed, USA); $\mathrm{SaO}_{2}$ Oxygen saturation; $t c \mathrm{CO}_{2}$ Transcutaneous carbon dioxide

adults with OSA with excellent sensitivity and specificity (19-21,25), with one study (19) reporting a sensitivity of $91 \%$ and specificity of $95 \%$ for subjects with a diagnosis of OSA (AHI $>15$ ). The possible reasons for lower specificity in the current study compared with the adult study may be explained by a lower incidence of moderate to severe AHI in the current group; the tendency for children to experience less significant hypoxemia than adults, making it more difficult to delineate events in the absence of oxygen desaturations; the inability of the device to distinguish central versus obstructive apneas; and mouth breathing in children that may be mistaken for respiratory events with reduced airflow. A more recent study examining the utility of the multichannel AL device in obese adolescents (mean age 13 years) observed SDB in 19 of 25 subjects with a sensitivity and specificity of $>80 \%$ using an obstructive AHI cut-off value $>5$ events/h (22). The reason for improved specificity in that study may be explained by the fact that obese adolescents represent a more homogenous population than the spectrum of patients that present to a pediatric sleep program with SDB. We also found that, compared with the PSG data, the PSS-AL overestimated the AHI, similar to Lesser et al (22), and incorrectly diagnosed patients as having SDB. Possible reasons for the overestimation of the AHI resulting in 'false positives' include events scored during wakefulness, reduced flow observed on a nasal flow channel during mouth breathing, even in the absence of apnea and/or postarousal artefacts mistaken as reduced airflow. The manual and automated PSS-AL scoring were comparable in diagnosing moderate to severe SDB when present but comparably misclassified the severity of SDB compared with PSG.

The limitations of the present study warrant consideration. The sample population included children who were referred to a tertiary pediatric sleep centre, which may limit the generalizability of these results to a community-based, nonreferred sample. We deliberately chose to include a spectrum of patients who were likely to present to pediatric programs to address the utility of such a device within a pediatric sleep facility. Moreover, there were no baseline or PSG differences between patients with comorbidities and healthy children in this group.

The PSS-AL cannot distinguish between central and obstructive apneas, which, together with the lack of electroencephalogram data, may result in misclassification of subjects. Additional limitations of portable monitors include missing data due to lack of application, malfunction and intolerance of the nasal cannula. The data loss 
TABLE 2

The apnea-hypopnea index (AHI) in subjects diagnosed using polysomnography and the PSS-AL monitor

\begin{tabular}{lcc}
\hline SDB according to the type of sleep study & Polysomnography & Manual PSS-AL \\
\hline $\mathrm{AHI}<1.5$ events/h & $18(51)$ & $4(11)$ \\
Mild SDB (AHI $\geq 1.5$ events/h to $<5$ events/h) & $12(34)$ & $8(23)$ \\
Moderate SDB $(\mathrm{AHI} \geq 5$ events/h to $<10$ events/h) & $1(4)$ & $12(34)$ \\
Severe SDB $(\mathrm{AHI} \geq 10$ events/h) & $4(11)$ & $11(32)$ \\
\hline
\end{tabular}

Data presented as $n$ (\%). PSS Portable sleep study; PSS-AL Portable sleep study-ApneaLink (ResMed, USA); SDB Sleep disordered breathing

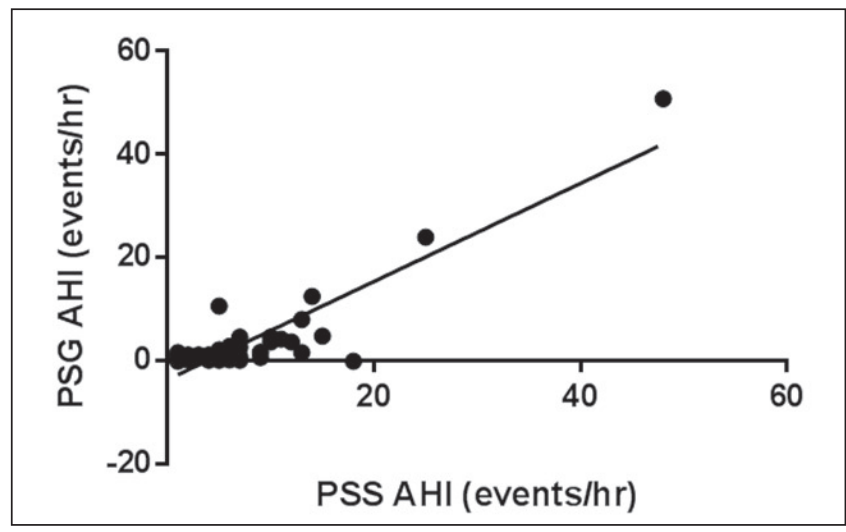

Figure 1) Correlation comparing manual scoring of apnea-hypopnea index (AHI) using the portable sleep study-ApneaLink (PSS-AL, ResMed, USA) monitor and polysomnography AHI

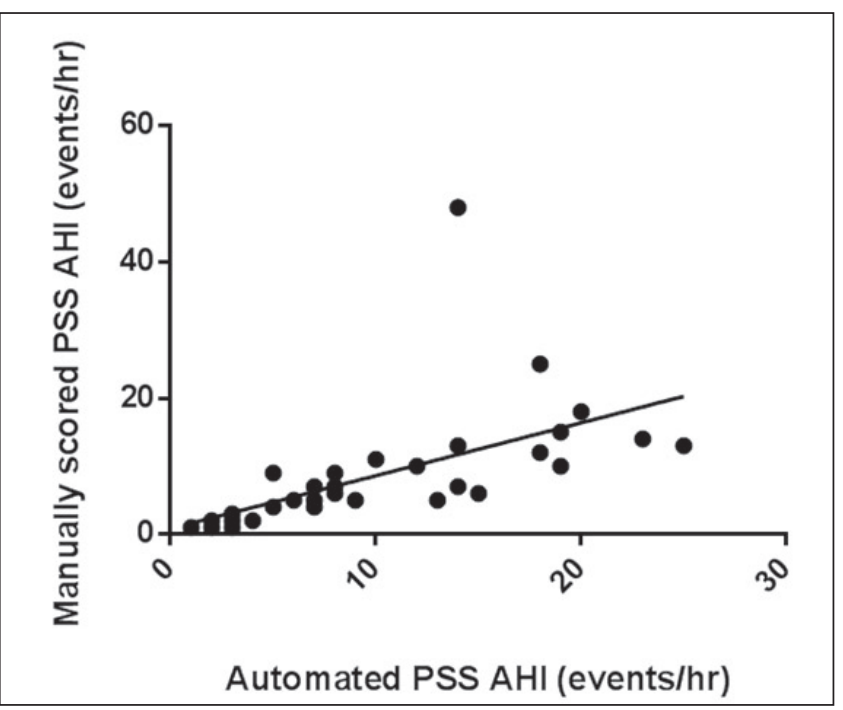

Figure 2) Correlation between manual and automated apnea-hypopnea index (AHI) scoring using the portable sleep study (PSS)-ApneaLink (ResMed, USA) monitor

reported in our study is comparable with that reported in other studies $(19,22)$. Furthermore, the PSS-AL monitor was not used in a home setting; therefore, we are unable to determine its reliability and

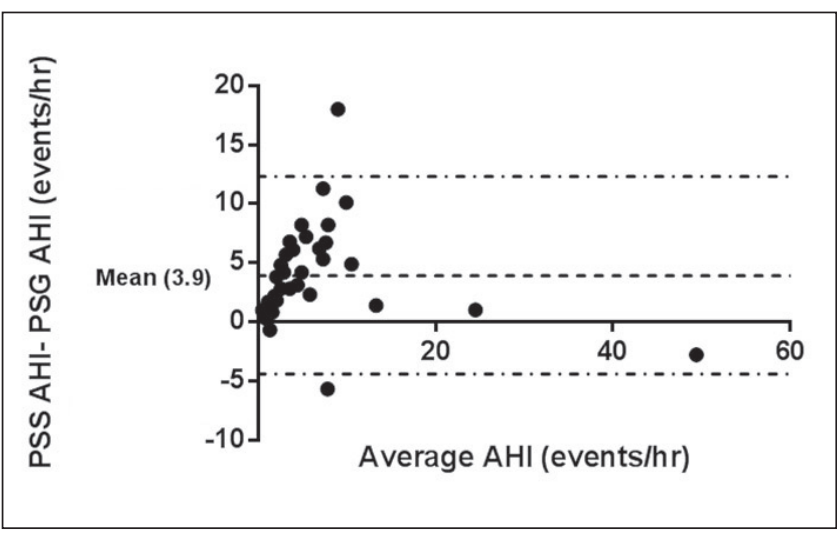

Figure 3) Bland-Altman plot showing the difference between manually scored portable sleep study-ApneaLink (PSS-AL, ResMed, USA) apneahypopnea index (AHI) and the polysomnography (PSG) AHI against the mean of the AHI values. The mean difference is \pm 3.9 events events/h. There are two outliers with a mean difference $> \pm 2$ SDs. One subject was awake for a significant proportion of the study, causing an overestimation of his AHI on the PSS-AL. The second outlier was a subject with sickle-cell disease who almost exclusively experienced hypopneas related to arousals rather than desaturations, accounting for the lower PSS-AL AHI (5.0 events/h) compared with PSG AHI (10.4 events/h). Dashed line represents \pm 2 SDs

feasibility, although this has been reported in the adult literature $(19,26)$. During the study, there were no adverse events from using this tool in the sleep laboratory. Because we did not study children $<4$ years of age, we are unable to comment on the tolerability and safety of these tools in very young children.

A PSG test is labour intensive, time consuming, expensive and can be particularly difficult to perform in children. Due to the ease of use and relatively inexpensive operating costs, portable sleep monitors offer promise as a screening tool for children to readdress the balance of inequity in the timely diagnosis and management of SDB. However, these portable monitors, given the listed limitations, should be used in the context of a pediatric sleep program with clinical evaluation for management of these patients.

\section{CONCLUSION}

The lack of timely diagnosis of sleep disorders in children limits the scope of potentially beneficial treatments. Given the long wait times for PSG, the single-channel AL monitor is a useful screening tool for triage in pediatric sleep laboratories, although it cannot and should

TABLE 3

Sensitivities and specificities of automated and manually scored PSS-AL AHI compared with PSG AHI

\begin{tabular}{|c|c|c|c|c|c|c|c|c|}
\hline \multirow[b]{2}{*}{ PSS-AL AHI } & \multicolumn{4}{|c|}{ PSG SDB (AHI $\geq 1.5$ events/h) } & \multicolumn{4}{|c|}{ PSG SDB (AHI $\geq 5$ events/h) } \\
\hline & Sensitivity & Specificity & PPV & NPV & Sensitivity & Specificity & PPV & NPV \\
\hline Manual PSS-AL (cut-off $\geq 1.5$ events/h) & 94 & 16 & 51 & 75 & 100 & 13 & 16 & 100 \\
\hline Manual PSS-AL (cut-off $\geq 5$ events/h) & 94 & 61 & 70 & 92 & 100 & 40 & 22 & 100 \\
\hline Automated PSS-AL (cut-off $\geq 5$ events/h) & 94 & 44 & 62 & 88 & 100 & 30 & 19 & 100 \\
\hline
\end{tabular}

Data presented as \%. AHI Apnea-hypopnea index; NPV Negative predictive value; PSG Polysomnography; PSS-AL Portable sleep study-ApneaLink (ResMed, USA); PPV Positive predictive value; SDB Sleep disordered breathing 
not replace PSG. We recommend its use only in the context of an appropriate clinical evaluation. Future research is urgently needed to address the role of portable sleep monitors in home settings of otherwise healthy children in the community.

ACKNOWLEDGEMENTS: The authors are grateful to the children and families who participated in this study.

DISCLOSURES: This study was unfunded and the authors have no financial disclosures or conflicts of interest to declare.

\section{REFERENCES}

1. Lumeng JC, Chervin RD. Epidemiology of pediatric obstructive sleep apnea. Proc Am Thorac Soc 2008;5:242-52.

2. Verhulst SL, Van Gaal L, De Backer W, Desager K. The prevalence, anatomical correlates and treatment of sleep-disordered breathing in obese children and adolescents. Sleep Med Rev 2008;12:339-46.

3. Kritzinger FE, Al-Saleh S, Narang I. Descriptive analysis of central sleep apnea in childhood at a single center. Pediatr Pulmonol 2011;46:1023-30

4. Iber C, Medicine AAoS. The AASM Manual for the Scoring of Sleep and Associated Events: Rules, Terminology and Technical Specifications: American Academy of Sleep Medicine; 2007.

5. Wise MS, Nichols CD, Grigg-Damberger MM, et al. Executive summary of respiratory indications for polysomnography in children: An evidence-based review. Sleep 2011;34:389-98AW.

6. Capdevila OS, Kheirandish-Gozal L, Dayyat E, Gozal D. Pediatric obstructive sleep apnea: Complications, management, and longterm outcomes. Proc Am Thorac Soc 2008;5:274-82.

7. Gozal D, Kheirandish-Gozal L, Bhattacharjee R, Spruyt K. Neurocognitive and endothelial dysfunction in children with obstructive sleep apnea. Pediatrics 2010;126:e1161-7.

8. Owens JA. Neurocognitive and behavioral impact of sleep disordered breathing in children. Pediatr Pulmonol 2009;44:417-22.

9. Randhawa PS, Cetto R, Chilvers G, Georgalas C, Narula AA. Long-term quality-of-life outcomes in children undergoing adenotonsillectomy for obstructive sleep apnoea: A longitudinal study. Clin Otolaryngol 2011;36:475-81.

10. Baugh RF, Archer SM, Mitchell RB, et al. Clinical practice guideline: Tonsillectomy in children. Otolaryngol Head Neck Surg 2011;144(1 Suppl):S1-30.

11. Brown KA, Morin I, Hickey C, Manoukian JJ, Nixon GM, Brouillette RT. Urgent adenotonsillectomy: An analysis of risk factors associated with postoperative respiratory morbidity. Anesthesiology 2003;99:586-95.

12. Raghavendran S, Bagry H, Detheux G, Zhang X, Brouillette RT, Brown KA. An anesthetic management protocol to decrease respiratory complications after adenotonsillectomy in children with severe sleep apnea. Anesth Analg 2010;110:1093-101.

13. Flemons WW, Douglas NJ, Kuna ST, Rodenstein DO, Wheatley J. Access to diagnosis and treatment of patients with suspected sleep apnea. Am J Respir Crit Care Med 2004;169:668-72.

14. Chervin RD, Weatherly RA, Garetz SL, et al. Pediatric sleep questionnaire: Prediction of sleep apnea and outcomes. Arch Otolaryngol Head Neck Surg 2007;133:216-22.

15. Nixon GM, Brouillette RT. Diagnostic techniques for obstructive sleep apnoea: Is polysomnography necessary? Paediatr Respir Rev 2002;3:18-24.

16. Chau KW, Ng DK, Kwok KL, et al. Application of videotape in the screening of obstructive sleep apnea in children. Sleep Med 2008;9:442-5.

17. Brouillette RT, Lavergne J, Leimanis A, Nixon GM, Ladan S, McGregor CD. Differences in pulse oximetry technology can affect detection of sleep-disorderd breathing in children. Anesth Analg 2002;94(1 Suppl):S47-53.

18. Marcus CL, Brooks LJ, Draper KA, et al. Diagnosis and management of childhood obstructive sleep apnea syndrome. Pediatrics 2012;130:e714-55.

19. Erman MK, Stewart D, Einhorn D, Gordon N, Casal E. Validation of the ApneaLink for the screening of sleep apnea: A novel and simple single-channel recording device. J Clin Sleep Med 2007;3:387-92.

20. Ng SS, Chan TO, To KW, et al. Validation of a portable recording device (ApneaLink) for identifying patients with suspected obstructive sleep apnoea syndrome. Intern Med J 2009;39:757-62.

21. Oktay B, Rice TB, Atwood CW Jr, et al. Evaluation of a singlechannel portable monitor for the diagnosis of obstructive sleep apnea. J Clin Sleep Med 2011;7:384-90.

22. Lesser DJ, Haddad GG, Bush RA, Pian MS. The utility of a portable recording device for screening of obstructive sleep apnea in obese adolescents. J Clin Sleep Med 2012;8:271-7.

23. Iber C A-IS, Chesson A, Quan SF; for the American Academy of Sleep Medicine. The AASM Manual for the Scoring of Sleep and Associated Events: Rules, Terminology and Technical Specifications. Winchester: American Academy of Sleep Medicine, 2007.

24. Marcus CL, Omlin KJ, Basinki DJ, et al. Normal polysomnographic values for children and adolescents. Am Rev Respir Dis 1992;146(5 Pt 1):1235-9.

25. Chen H, Lowe AA, Bai Y, Hamilton P, Fleetham JA, Almeida FR. Evaluation of a portable recording device (ApneaLink) for case selection of obstructive sleep apnea. Sleep Breath 2009;13:213-9.

26. Clark AL, Crabbe S, Aziz A, Reddy P, Greenstone M. Use of a screening tool for detection of sleep-disordered breathing. J Laryngol Otol 2009;123:746-9. 


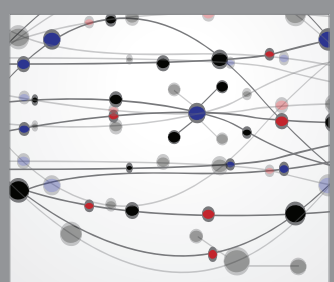

The Scientific World Journal
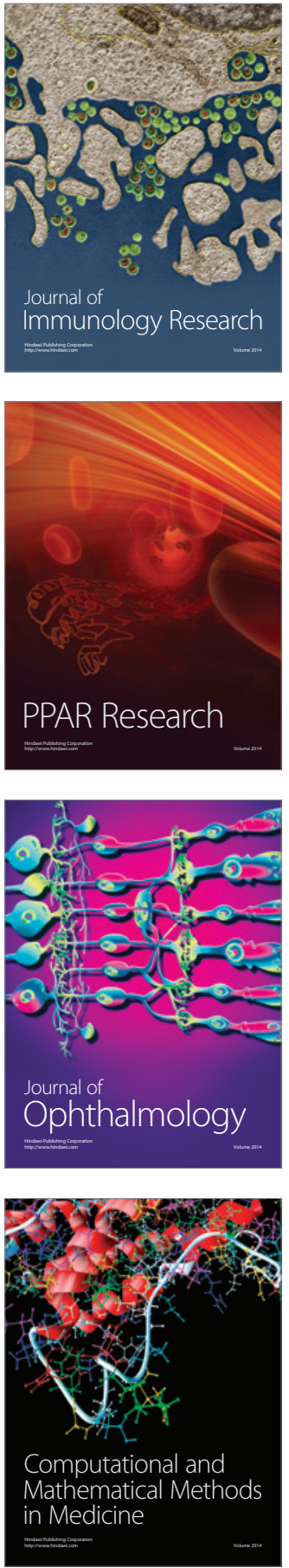

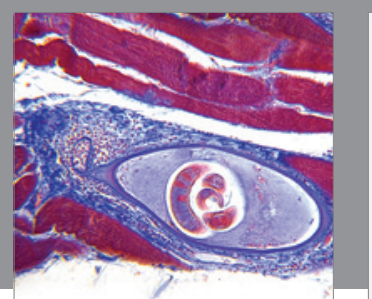

Gastroenterology Research and Practice

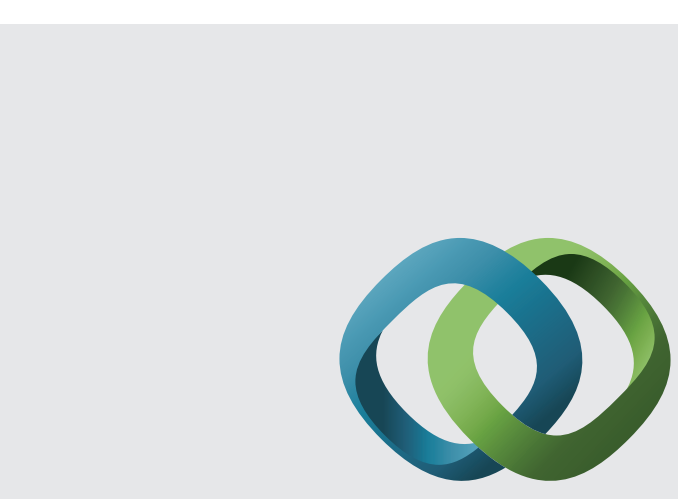

\section{Hindawi}

Submit your manuscripts at

http://www.hindawi.com
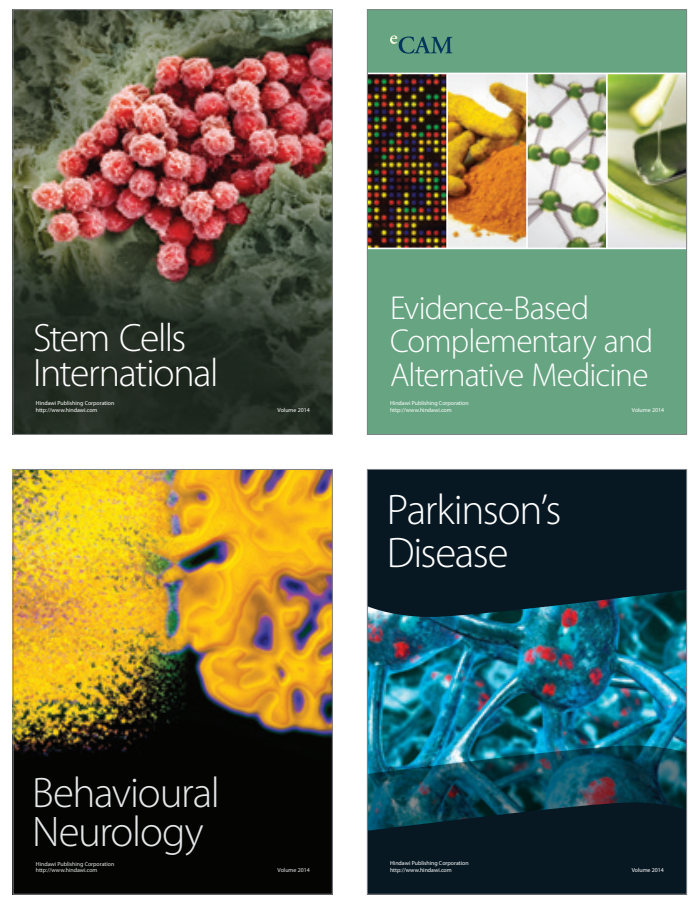
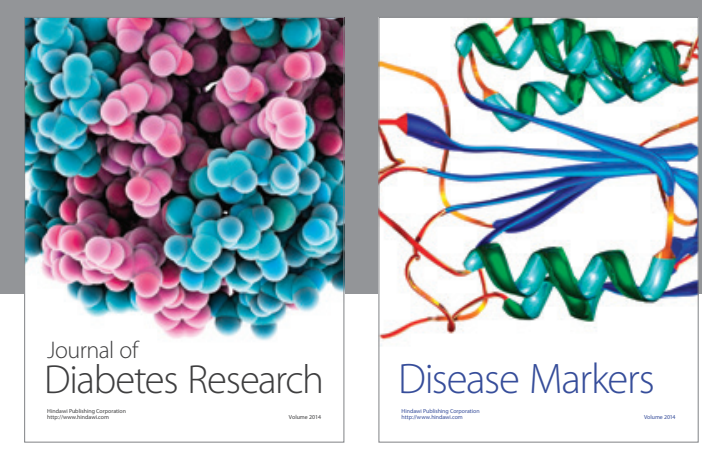

Disease Markers
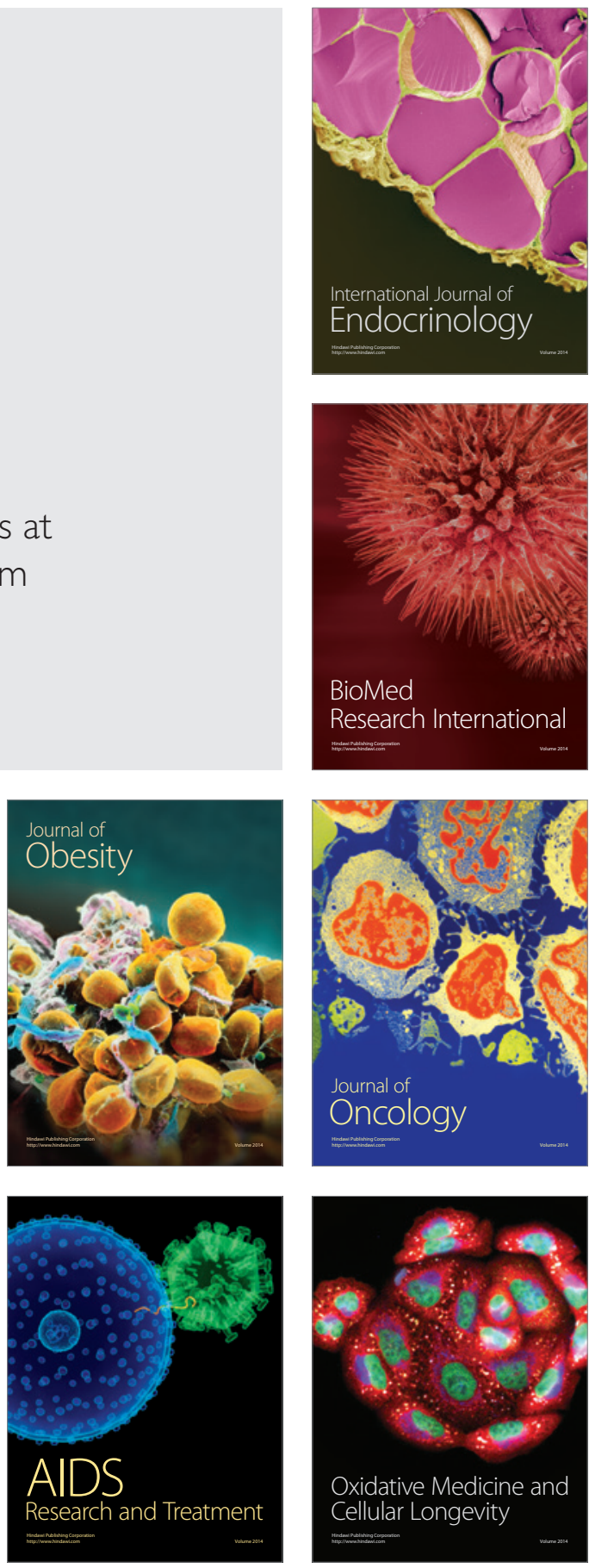